\title{
Qualitative properties of some discrete models of disease propagation
}

\author{
István Faragó ${ }^{\mathrm{a}, \mathrm{b}}$, Róbert Horváth ${ }^{\mathrm{c}, \mathrm{b}, *}$ \\ a Department of Applied Analysis and Computational Mathematics, Eötvös Loránd University, Pázmány Péter stny. 1/C, 1117, Budapest, \\ Hungary \\ ${ }^{\mathrm{b}}$ MTA-ELTE NumNet Research Group, Hungary \\ ${ }^{c}$ Department of Analysis, Budapest University of Technology and Economics, Egry J. u. 1. 1111, Budapest, Hungary
}

\section{A R T I C L E I N F O}

\section{Article history:}

Received 31 May 2017

Received in revised form 6 September 2017

\section{Keywords:}

Epidemic models

Qualitative properties of systems of pdes

Nonnegativity

Finite difference method

\begin{abstract}
A B S T R A C T
In this paper the qualitative properties of certain spatial disease propagation models are investigated. The paper can be considered as a generalization of the papers (Faragóand Horváth, 2016; 2017). The models of these papers assume that the members of the population do not move and that the infection is localized in the sense that only the members in a certain neighbourhood of the infective member can be infected. The considered qualitative properties were: the nonnegativity and the monotonicity of the density functions, and the preservation of the amount of the members. Sufficient conditions for these properties were obtained for the mesh size and the time step in certain finite difference solutions of the model. In these works, the one-dimensional case was investigated only. The present paper extends the above result in two directions: with results for higher dimensional problems and for another disease propagation model given in Capasso (2008). Here the members are allowed to move according to some diffusion law. Similarly to the previous model, sufficient conditions are given that guarantee the validity of the qualitative properties. We focus only on the properties of the discrete models. The results are verified on test problems.
\end{abstract}

(C) 2017 Elsevier B.V. All rights reserved.

\section{Introduction}

Mathematical models are efficient tools of modelling disease propagation. In the most popular compartmental models, the population is considered to be homogeneously mixed and the individuals are classified according to their relation to the disease. Thus, the population is divided into some disjoint groups, so-called compartments, and the change of the number of individuals in the compartments is described in the form of a system of ordinary differential equations. The three most typical compartments are as follows: susceptibles (who have yet to contract the disease and become infectious), infectives (who can pass on the disease to others) and removed (who have been infected but cannot transmit the disease for some reason). Extending this compartment system with other appropriate compartments and defining the appropriate dynamics between them, the model gives realistic results that can be used for example in public health considerations (hygiene, vaccination, quarantine, etc.) [1-5].

The main drawback of the compartmental models is that it gives only results for the number of the members in the compartments but not for the spatial location of them. We know from the devastating pandemics of the human history (e.g. black death, Spanish flu) that the place of the infective focus and its speed is a very important piece of information

\footnotetext{
* Corresponding author at: Department of Analysis, Budapest University of Technology and Economics, Egry J. u. 1. 1111, Budapest, Hungary.

E-mail addresses: faragois@cs.elte.hu (I. Faragó), rhorvath@math.bme.hu (R. Horváth).
} 
in the prevention of the outbreak of an epidemic. This is why it is a natural expectation to construct and investigate spacedependent mathematical models. If we do not want to leave the field of ordinary differential equations, we can set up the socalled meta-population models. Here the original population is divided into subpopulations according to some geopolitical considerations and are connected somehow into a network by some prescribed rules for the disease propagation [2]. This method results in a system of ordinary differential equations. Another possibility is to apply partial differential equation models.

In this paper we investigate two types of partial differential equation models of disease propagation: the local spatial SIR model and the diffusive spatial SIR model. Both models come from the so-called SIR model [3,5]

$$
\begin{aligned}
S^{\prime} & =-a S I, \\
I^{\prime} & =a S I-b I, \\
R^{\prime} & =b I,
\end{aligned}
$$

where $S=S(t), I=I(t)$ and $R=R(t)$ denote, respectively, the number of susceptible, infective and recovered individuals as a function of time $t$. The contact rate $a$ and recovery coefficient $b(1 / b$ is the duration of the average infectious period) are positive known numbers. In this model there is no vital dynamics (birth and death are neglected) and the recovered individuals do not become susceptible again.

The local spatial SIR model (IsSIR). In the local spatial SIR model, based on Kendall's suggestion [6], we suppose that the individuals do not move and that the infection is local and related to the distance between them, that is an individual can be infected only by others located in a certain neighbourhood. This model is especially appropriate for the study of plant diseases but similar models can be applied also for modelling of disease transmission in human populations [7-9]. We suppose that the functions $S, I$ and $R$ depend not only on the time variable $t$ but also on the spatial coordinate $x \in \mathbb{R}^{D}$, where $D$ is the spatial dimension of the problem. Thus, from now on, the functions $S=S(x, t), I=I(x, t)$ and $R=R(x, t)$ denote the density of susceptibles, infectives and recovered, respectively. Thus, the integral of these density functions on a certain spatial domain gives the number of the members located in that domain.

In order to take into the account the localized character of the disease propagation, we change the product $a S$ in (1) to the integral

$$
\int_{K(x)} W\left(\left|x^{\prime}-x\right|\right) I\left(x^{\prime}, t\right) \mathrm{d} x^{\prime}
$$

This integral describes how the infectives at the points $x^{\prime}$ affect the infection of the susceptible at the point $x$. The nonnegative weighting function $W$ is supposed to depend only on the Euclidean distance of the points $x^{\prime}$ and $x$, and $K(x)$ denotes a prescribed neighbourhood of the point $x$. With the above modification we arrive at the system of integro-differential equations

$$
\begin{aligned}
S_{t}^{\prime}(x, t) & =-\left(\int_{K(x)} W\left(\left|x^{\prime}-x\right|\right) I\left(x^{\prime}, t\right) \mathrm{d} x^{\prime}\right) S(x, t), \\
I_{t}^{\prime}(x, t) & =\left(\int_{K(x)} W\left(\left|x^{\prime}-x\right|\right) I\left(x^{\prime}, t\right) \mathrm{d} x^{\prime}\right) S(x, t)-b I(x, t), \\
R_{t}^{\prime}(x, t) & =b I(x, t) .
\end{aligned}
$$

We can approximate the above system with a system of partial differential equations. In order to keep this approximation traceable, we suppose that $K(x)$ is a closed ball around $x$ with radius $\delta$ :

$$
K(x)=\left\{x^{\prime} \in \mathbb{R}^{D}|| x^{\prime}-x \mid \leq \delta\right\} .
$$

We develop the function $I$ into Taylor series at the spatial point $x$, and we approximate $I\left(x^{\prime}, t\right)$ with the second order Taylor polynomial. In this way, we arrive at the system

$$
\begin{aligned}
S_{t}^{\prime} & =-S\left(\vartheta I+\varphi \Delta_{D} I\right), \\
I_{t}^{\prime} & =S\left(\vartheta I+\varphi \Delta_{D} I\right)-b I, \\
R_{t}^{\prime} & =b I,
\end{aligned}
$$

where $\Delta_{D}$ is the $D$ dimensional spatial Laplace operator, and

$$
\vartheta=\int_{K(0)} W(|u|) \mathrm{d} u, \varphi=\frac{1}{2} \int_{K(0)} u_{1}^{2} W(|u|) \mathrm{d} u
$$

$\left(u=\left(u_{1}, \ldots, u_{D}\right)=x^{\prime}-x \in K(0)\right)$ are positive constants that are determined by the weighting function $W$. A natural choice for the weighting function on $K(x)$ is $W(u)=\max \{0,1-u / \delta\}$ (naturally, the graph for negative $u$ values is irrelevant). We can also use some positive multiple of this function. In real life modelling, the most important cases are dimensions $D=1$ and $D=2$. For these cases, the $\vartheta$ and $\varphi$ values can be calculated easily and can be found in Table 1 .

The derivation of (4) for the one-dimensional case can be found in [4]. The validity of (4) for higher dimensions will be proved in the next section (cf. Theorem 1). 
Table 1

The $\vartheta$ and $\varphi$ values in $D=1$ and $D=2$ spatial dimensions.

\begin{tabular}{lll}
\hline & $\vartheta$ & $\varphi$ \\
\hline$D=1$ & $\delta$ & $\delta^{3} / 12$ \\
$D=2$ & $\delta^{2} \pi / 3$ & $\delta^{4} \pi / 40$ \\
\hline
\end{tabular}

System (4) must be equipped with some initial and boundary conditions. Albeit there is no spatial movement in the present model, we need boundary conditions because of the spatial derivatives of second order. We have chosen to use homogeneous Dirichlet boundary condition to model the situation when the environmental conditions are incompatible with life outside the considered domain of habitat.

The diffusive spatial SIR model (dsSIR). The diffusive spatial SIR model can be obtained from the SIR model (1) by simply adding diffusion terms to each equation and by making the unknown functions space dependent. Thus, we arrive at the system of partial differential equations

$$
\begin{aligned}
S_{t}^{\prime}(x, t) & =d_{S} \Delta_{D} S(x, t)-k I(x, t) S(x, t), \\
I_{t}^{\prime}(x, t) & =d_{I} \Delta_{D} I(x, t)+k I(x, t) S(x, t)-b I(x, t), \\
R_{t}^{\prime}(x, t) & =d_{R} \Delta_{D} R(x, t)+b I(x, t),
\end{aligned}
$$

where $k$ is a positive parameter and $d_{S}, d_{I}$ and $d_{R}$ are the diffusion coefficients of the $S, I$ and $R$ subpopulations, respectively [3].

Remark 1. The main characteristic difference between the two systems (4) and (6) is in their first equations, which are formulated for $S(x, t)$. In system (6), the flux of the susceptibles is proportional with $\nabla_{D} S$ with constant diffusion coefficient $d_{S}$, while in system (4) the flux is proportional with $\nabla_{D} I$ with the variable coefficient $-\varphi S$.

For ordinary differential equation models the number and the type of the equilibria are generally investigated, while for partial differential equation models travelling waves or nontrivial epidemic states are sought. However, only a little attention is devoted to obtaining qualitatively adequate solutions of different continuous and discrete models (e.g. [10,11]).

In this paper, we will investigate the qualitative properties of the systems (4) and (6) and some consistent finite difference discretizations of them. In Section 2, we investigate the IsSIR model from qualitative point of view, while in Section 3 similar analysis is done for the dsSIR model. In both cases a priori upper bounds are calculated for the finite difference time step that guarantees the expected qualitative properties. The results are demonstrated on numerical test problems in both one and two dimensions. The results of the present paper can be considered as the generalization of the papers [12,13]. In these papers only the model (4) was investigated and only in the one spatial dimensional case.

\section{Investigation of the local spatial SIR model}

\subsection{Properties of the continuous model}

For one-dimensional problems, the simplified system (4) was deduced from the system of integro-differential equations (3) by using Taylor series expansion [4]. Before we turn to the investigation of the qualitative properties of the model, we prove that (3) simplifies to (4) also in higher spatial dimensions.

Theorem 1. Let us suppose that the second order spatial partial derivatives of I are continuous, the weighting function is $W(u)=\max \{0,1-u / \delta\}$, and the $K(x)$ neighbourhoods are closed balls. Then approximating $I$ with its second order spatial Taylor polynomial, system (3) simplifies to the system (4).

Proof. The proof can be carried out easily using the special form of the weighting function $W$ and the symmetry of the neighbourhood $K(0)$.

The disease propagation models must satisfy some characteristic qualitative properties of the real disease propagation process. Because spatial motion is not allowed in the lsSIR model and it does not contain terms responsible for birth and death, the sum of the three population densities has to remain constant at every spatial point $x$. This is the so-called local mass conservation property:

[P1] (Local mass conservation property)

$$
S(x, t)+I(x, t)+R(x, t)=S(x, 0)+I(x, 0)+R(x, 0)=: N(x), \forall t \geq 0 .
$$

This property is trivially valid for the system (4). To see this we have to add only the three equations. Functions $S, I$ and $R$ denote population densities, thus it is natural to require that these functions are nonnegative. This is the so-called nonnegativity property: 
[P2] (Nonnegativity property) Condition $S(x, 0), I(x, 0), R(x, 0) \geq 0$ for all $x$ implies $S(x, t), I(x, t), R(x, t) \geq 0$ for all $x$ and for all $t>0$.

The members of the removed compartment do not become susceptible again. Thus, we can conclude that the density of the susceptibles cannot grow and the density of the removed cannot decrease in time. This is the so-called monotonicity property:

[P3] (Monotonicity property) $S\left(x, t_{2}\right) \leq S\left(x, t_{1}\right)$ and $R\left(x, t_{2}\right) \geq R\left(x, t_{1}\right)$ for all $0 \leq t_{1}<t_{2}$ and fixed $x$.

Similarly to the one-dimensional case (see [12]), we are not able to give a priori conditions for the continuous system (4) that guarantee the above properties P1-P3. An a posteriori condition can be formulated as follows. If the condition $p(x, t):=\vartheta I(x, t)+\varphi \Delta_{D} I(x, t) \geq 0$ is satisfied for all $x$ and for all $t \geq 0$, then properties P1-P3 are true for the solution of problem (4). In the contrary to the continuous case, we will see later, that a priori conditions can be obtained in the discrete case.

Remark 2. We notice that with the notation $p(x, t):=\vartheta I(x, t)+\varphi \Delta_{D} I(x, t)$, system (4) can be rewritten in the form

$$
\begin{aligned}
S_{t}^{\prime} & =-S p, \\
I_{t}^{\prime} & =S p-b I, \\
R_{t}^{\prime} & =b I,
\end{aligned}
$$

and the time derivative of $p$ has the form $p_{t}^{\prime}=(\vartheta S-b) p+\varphi \Delta_{D}(S p)$. This formula will be behind the discretizations and the estimations in the formulas (14) and (19).

Travelling wave solutions mimic epidemic. These solutions of system (4) can be sought and investigated similarly to the one-dimensional case [4,12]. We can search for plane wave solutions in the form

$$
S(x, t)=\tilde{S}(n \cdot x-c t), \quad I(x, t)=\tilde{I}(n \cdot x-c t), \quad R(x, t)=\tilde{R}(n \cdot x-c t),
$$

where $c$ is a constant that denotes the wave speed, and $n$ is the unit vector in the direction of the wave propagation. The univariate functions $\tilde{I}$ and $\tilde{S}$ have the properties

$$
\lim _{\xi \rightarrow \pm \infty} \tilde{I}(\xi)=0, \lim _{\xi \rightarrow \pm \infty} \tilde{I}^{\prime}(\xi)=0, \lim _{\xi \rightarrow \infty} \tilde{S}(\xi)=: \tilde{S}^{\infty}>0 .
$$

These expressions formulate the fact that there are no infected members at the time instants $t= \pm \infty$ and the density of the susceptible members is a positive constant at the time instant $t=-\infty$.

Inserting functions (8) into the system (4), integrating the equations from $\xi$ to $\infty$ and taking into the consideration the assumptions (9), we arrive at a system of ordinary differential equations for the functions $\tilde{S}, \tilde{I}$ and $\tilde{R}$. It can be shown with the investigation of this system, that a necessary condition of a wave form solution, that is for an epidemic, is

$$
\tilde{S}^{\infty}>b / \vartheta,
$$

which means that the initial density of the susceptible subpopulation must be sufficiently large.

\subsection{Properties of the discrete models}

In this section we derive conditions that guarantee the discrete analogues of the qualitative properties P1-P3 (also denoted by P1-P3) for some finite difference numerical solutions of the system (4). We will consider the explicit Euler (EE) method and a version of an implicit-explicit (IMEX) method.

First we construct the spatial mesh then we formulate the EE and the IMEX methods. We solve the problem on the cube $[0, L]^{D}$. Homogeneous Dirichlet boundary condition is applied on the boundary. We define a uniform spatial grid

$$
\begin{aligned}
\omega_{h}= & \left\{\left(x_{1}, \ldots, x_{D}\right) \in[0, L]^{D} \mid x_{k} \in\{0, h, \ldots,(M+1) h\}, k=1, \ldots, D,\right. \\
& \left.h=L /(M+1), M \in \mathbb{N}^{+}\right\}
\end{aligned}
$$

and a positive time step $\tau>0$. The functions $S, I$ and $R$ are approximated, respectively, by the grid functions $S^{n}, I^{n}$ and $R^{n}$ at the $n$th time level $t=n \tau$. Thus, e.g. $S_{1, \ldots, 1}^{n}$ approximates $S(h, \ldots, h, n \tau)$. For $n=0$, the values of the grid functions are known from the initial conditions, moreover the values are equal to zero in the boundary points. We have to calculate the values of the grid function in the inner points of the grid. In the usual way, we apply the one-parameter-indexing of the components of the grid functions. With the column-wise indexing we reshape the values of the grid functions into column vectors, denoted by $s^{n}, i^{n}$ and $r^{n}$, respectively. These vectors are from the space $\mathbb{R}^{M^{D}}$. In this way we obtain that the value $S(h, \ldots, h, n \tau)$ in the above example will be approximated by the value $s_{M}^{1}$.

In order to simplify the notations, we will use the following conventions: relations are meant element-by-element, thus $A \geq B$ means $a_{i j} \geq b_{i j}$ and $A \leq 1$ means $a_{i j} \leq 1$. With a scalar value $\alpha$ we define $(\alpha+A)_{i j}=\alpha+a_{i j}$, moreover we frequently apply the element-by-element product of matrices - the so-called Hadamard product - $(A \circ B)_{i j}=a_{i j} b_{i j}$. 
With the above notations, the explicit Euler scheme for the system (4) can be written as follows.

$$
\begin{aligned}
& \frac{s^{n+1}-s^{n}}{\tau}=-s^{n} \circ p^{n}, \\
& \frac{i^{n+1}-i^{n}}{\tau}=s^{n} \circ p^{n}-b i^{n}, \\
& \frac{r^{n+1}-r^{n}}{\tau}=b i^{n},
\end{aligned}
$$

where $p^{n}=\vartheta i^{n}+\left(\varphi / h^{2}\right) Q_{D} i^{n}$ and $Q_{D} / h^{2}$ is the discretization matrix of the $D$ dimensional Laplace operator $\Delta_{D}$. Let us define the tridiagonal matrix $Q=\operatorname{tridiag}(1,-2,1) \in \mathbb{R}^{M}$. Then, if $D=1$ then $Q_{D}=Q$ and in the case of $D=2$ we have $Q_{D}=I_{M} \otimes Q+Q \otimes I_{M}$, where $I_{M} \in \mathbb{R}^{M \times M}$ is the identity matrix and $\otimes$ denotes the Kronecker product.

For the EE scheme (11) the qualitative properties can be guaranteed by an a priori condition.

Theorem 2. Let us suppose that at the initial state $s^{0} \geq 0, i^{0} \geq 0, r^{0} \geq 0$, and $p^{0} \geq 0$, moreover assume that

$$
\tau \leq \min \left\{\frac{1}{b+2 D \varphi N_{\max } / h^{2}}, \frac{1}{N_{\max }\left(\vartheta+2 D \varphi / h^{2}\right)}\right\},
$$

where $N_{\max }=\max \left(s^{0}+i^{0}+r^{0}\right)$. Then the EE finite difference scheme satisfies the qualitative properties P1-P3.

Proof. Property P1 is satisfied without any condition. This can be seen easily when we sum up the three equations in (11). To prove the properties P2-P3, we will use induction. First we show the following implication: for any $n \in \mathbb{N}$

$$
\begin{gathered}
s^{n} \geq 0, i^{n} \geq 0, r^{n} \geq 0, p^{n} \geq 0, \tau p^{n} \leq 1 \\
\Downarrow \\
s^{n} \geq s^{n+1} \geq 0, i^{n+1} \geq 0, r^{n+1} \geq r^{n} \geq 0, p^{n+1} \geq 0, \tau p^{n+1} \leq 1 .
\end{gathered}
$$

From the first equation of (11) we have $s^{n+1}=s^{n} \circ\left(1-\tau p^{n}\right)$. Because of $0 \leq \tau p^{n} \leq 1$, the condition for $s^{n+1}$ is satisfied. From the second equation $i^{n+1}=i^{n}+\tau s^{n} \circ p^{n}-\tau b i^{n}=(1-\tau b) i^{n}+\tau s^{n} \circ p^{n}$. In view of $(12), \tau$ is not greater than $1 / b$, thus $i^{n+1}$ is nonnegative indeed.

From the third equation we have $r^{n+1}=r^{n}+\tau b i^{n} \geq r^{n} \geq 0$. Thus, the condition for $r^{n+1}$ is also satisfied.

We show that $p^{n+1} \geq 0$. Due to the nonnegativity of the vector in $s^{n} \circ p^{n}$ we have $Q_{D}\left(s^{n} \circ p^{n}\right)=\left(\operatorname{diag}\left(Q_{D}\right)+\operatorname{offdiag}\left(Q_{D}\right)\right)\left(s^{n} \circ\right.$ $\left.p^{n}\right) \geq \operatorname{diag}\left(Q_{D}\right)\left(s^{n} \circ p^{n}\right)=-2 D\left(s^{n} \circ p^{n}\right)$. Thus, $p^{n+1}$ can be estimated as follows.

$$
\begin{aligned}
p^{n+1} & =\vartheta i^{n+1}+\frac{\varphi}{h^{2}} Q_{D} i^{n+1} \\
& =\vartheta\left(\tau s^{n} \circ p^{n}+(1-b \tau) i^{n}\right)+\frac{\varphi}{h^{2}} Q_{D}\left(\tau s^{n} \circ p^{n}+(1-b \tau) i^{n}\right) \\
& =(1-b \tau) \underbrace{\left(\vartheta i^{n}+\frac{\varphi}{h^{2}} Q_{D} i^{n}\right)}_{p^{n}}+\tau \vartheta s^{n} \circ p^{n}+\tau \frac{\varphi}{h^{2}} Q_{D}\left(s^{n} \circ p^{n}\right) \\
& =\left(1-b \tau+\tau \vartheta s^{n}\right) \circ p^{n}+\tau \frac{\varphi}{h^{2}} Q_{D}\left(s^{n} \circ p^{n}\right) \\
& \geq\left(1-b \tau+\tau \vartheta s^{n}-2 \tau \frac{\varphi D}{h^{2}} s^{n}\right) \circ p^{n} \\
& =\left(1-\tau\left(b-\vartheta s^{n}+2 \frac{\varphi D}{h^{2}} s^{n}\right)\right) \circ p^{n} .
\end{aligned}
$$

Due to the nonnegativity of $s^{n}, i^{n}$ and $r^{n}$ and in view of the validity of property $\mathrm{P} 1$, we have the estimate $0 \leq s^{n} \leq s^{n}+i^{n}+r^{n}=$ $s^{0}+i^{0}+r^{0}=N_{\max }$. Similarly $0 \leq i^{n} \leq N_{\max }$ is also true. To the nonnegativity of $p^{n+1}$ it is enough to show that

$$
\tau\left(b-\vartheta s^{n}+2 \frac{\varphi D}{h^{2}} s^{n}\right) \leq 1
$$

in the last equation of (14). We can obtain this estimate as follows

$$
\tau\left(b-\vartheta s^{n}+2 \frac{\varphi D}{h^{2}} s^{n}\right) \leq \tau\left(b+2 \frac{\varphi D}{h^{2}} s^{n}\right) \leq \tau\left(b+2 \frac{\varphi D}{h^{2}} N_{\max }\right) \leq 1,
$$

which follows from the first term on the right hand side of the assumption (12). 
Now we prove that $\tau p^{n+1} \leq 1$.

$$
\begin{aligned}
\tau p^{n+1} & =\tau\left(\vartheta i^{n+1}+\frac{\varphi}{h^{2}} Q_{D} i^{n+1}\right) \\
& \leq \tau\left(\vartheta i^{n+1}+\frac{\varphi}{h^{2}} \operatorname{offdiag}\left(Q_{D}\right) i^{n+1}\right) \\
& \leq \tau N_{\max }\left(\vartheta+\frac{2 D \varphi}{h^{2}}\right) \leq 1,
\end{aligned}
$$

which is true because of the second upper bound for $\tau$ in the assumption (12). Let us notice that here we used only the fact that $s^{n+1}, i^{n+1}$ and $r^{n+1}$ are nonnegative, the local mass conservation property P1 and the assumption (12). This implies that also the condition $\tau p^{0} \leq 1$ must be satisfied. In view of the nonnegativity assumptions of the theorem, the first row of the implication (13) is valid with $n=0$. Above we showed that the implication is true for any $n$, which proves the theorem by induction.

In the investigation of the qualitative properties of differential equations it is typical that implicit methods give less strict upper bounds for the time step than the explicit ones. This is why we turn to implicit schemes. More precisely, we give the conditions of the validity of the properties P1-P3 for the following implicit-explicit scheme for the system (4)

$$
\begin{aligned}
& \frac{s^{n+1}-s^{n}}{\tau}=-s^{n+1} \circ p^{n} \\
& \frac{i^{n+1}-i^{n}}{\tau}=s^{n+1} \circ p^{n}-b i^{n+1}, \\
& \frac{r^{n+1}-r^{n}}{\tau}=b i^{n+1} .
\end{aligned}
$$

Theorem 3. Let us suppose that at the initial state $s^{0} \geq 0, i^{0} \geq 0, r^{0} \geq 0$, and $p^{0} \geq 0$, moreover assume that

$$
\tau \leq \begin{cases}\left(\left(2 D \varphi / h^{2}-\vartheta\right) N_{\max }\right)^{-1}, & \text { if } h<h^{\star}, \\ \text { arbitrary, } & \text { if } h \geq h^{\star},\end{cases}
$$

where $h^{\star}=(2 D \varphi / \vartheta)^{1 / 2}$. Then the IMEX scheme (16) satisfies the qualitative properties P1-P3.

Proof. Property P1 is satisfied automatically. In order to prove properties P2-P3, we use again induction and prove the implication

$$
\begin{gathered}
s^{n} \geq 0, i^{n} \geq 0, r^{n} \geq 0, p^{n} \geq 0 \\
\Downarrow \\
s^{n} \geq s^{n+1} \geq 0, i^{n+1} \geq 0, r^{n+1} \geq r^{n} \geq 0, p^{n+1} \geq 0 .
\end{gathered}
$$

From the first equation in (16) we conclude that $s^{n+1}=s^{n}-\tau s^{n+1} \circ p^{n}$, that is $s^{n+1} \circ\left(1+\tau p^{n}\right)=s^{n}$, which shows the implication for $s^{n+1}$.

The second equation can be rewritten in the form $(1+\tau b) i^{n+1}=i^{n}+\tau s^{n+1} \circ p^{n}$, so the nonnegativity of $i^{n+1}$ is evident.

The form $r^{n+1}=r^{n}+\tau b i^{n+1}$ of the third equation shows the validity of the implication for $r^{n+1}$.

It is left only the justification of the condition $p^{n+1} \geq 0$. First we give a lower bound for $s^{n+1} \circ p^{n}$

$$
\begin{aligned}
& Q_{D}\left(s^{n+1} \circ p^{n}\right)=\left(\operatorname{diag}\left(Q_{D}\right)+\operatorname{offdiag}\left(Q_{D}\right)\right)\left(s^{n+1} \circ p^{n}\right) \\
& \geq \operatorname{diag}\left(Q_{D}\right)\left(s^{n+1} \circ p^{n}\right)=-2 D s^{n+1} \circ p^{n},
\end{aligned}
$$

then we estimate $p^{n+1}$ as follows.

$$
\begin{aligned}
p^{n+1} & =\vartheta i^{n+1}+\frac{\varphi}{h^{2}} Q_{D} i^{n+1} \\
& =\vartheta\left(\frac{\tau}{1+\tau b} s^{n+1} \circ p^{n}+\frac{1}{1+\tau b} i^{n}\right)+\frac{\varphi}{h^{2}} Q_{D}\left(\frac{\tau}{1+\tau b} s^{n+1} \circ p^{n}+\frac{1}{1+\tau b} i^{n}\right) \\
& =\frac{1}{1+\tau b} \underbrace{\left(\vartheta i^{n}+\frac{\varphi}{h^{2}} Q_{D} i^{n}\right)}_{p^{n}}+\frac{\tau}{1+\tau b}\left(\vartheta s^{n+1} \circ p^{n}+\frac{\varphi}{h^{2}} Q_{D}\left(s^{n+1} \circ p^{n}\right)\right) \\
& =\frac{1}{1+\tau b} p^{n}+\frac{\tau}{1+\tau b}\left(\vartheta s^{n+1} \circ p^{n}+\frac{\varphi}{h^{2}} Q\left(s^{n+1} \circ p^{n}\right)\right) \\
& \geq \frac{1}{1+\tau b}\left(1+\tau\left(\vartheta-\frac{2 \varphi D}{h^{2}}\right) s^{n+1}\right) \circ p^{n} .
\end{aligned}
$$


If $h \geq h^{\star}$ then the expression in the last parenthesis is nonnegative, thus $p^{n+1}$ is nonnegative. In the case of $h<h^{\star}$ the nonnegativity of $p^{n+1}$ is guaranteed by the condition $\tau \leq\left(\left(2 D \varphi / h^{2}-\vartheta\right) N_{\max }\right)^{-1}$, that is by (17). Thus the implication (18) has been proved, and this completes the proof.

Remark 3. Let us notice that the scheme (16) can be implemented easily. From the reformulated system

$$
\begin{aligned}
\left(1+\tau p^{n}\right) \circ s^{n+1} & =s^{n}, \\
(1+\tau b) \circ i^{n+1} & =i^{n}+\tau s^{n+1} \circ p^{n}, \\
r^{n+1} & =r^{n}+\tau b i^{n+1},
\end{aligned}
$$

the unknown vectors $s^{n+1}, i^{n+1}$ and $r^{n+1}$ can be computed, respectively. Then we compute the vector $p^{n+1}$. We do not need to solve systems of linear equations in this scheme.

\section{Investigation of the diffusive spatial SIR model}

In the IsSIR model, we neglected the motion of the individuals and we were able to guarantee the qualitative properties of the system (4) only with the a posteriori condition $\vartheta I+\varphi \Delta_{D} I \geq 0$. If it can be assumed that the individuals of the population move according to some diffusive rules, then the diffusive model (6) can be a possible choice. In this part we investigate the qualitative properties of this model in the bounded space domain $\Omega \subset \mathbb{R}^{D}$ (with sufficiently smooth boundary), equipped with homogeneous Neumann boundary conditions and with a nonnegative initial condition.

\subsection{Properties of the continuous model}

The problem (6) with homogeneous Neumann boundary conditions and some given initial conditions has unique solution (see e.g. [14], Theorem 14.2, p. 196), and we assume that it is sufficiently smooth. Next, we formulate and show the validity of the main qualitative properties.

Unlike in the lsSIR model, in the dsSIR model the required qualitative properties cannot hold locally because of the motion of the individuals. In this case the qualitative properties will be understood for the whole space domain, that is for the functions

$$
\begin{aligned}
\tilde{S}(t) & =\int_{\Omega} S(x, t) \mathrm{d} x \\
\tilde{I}(t) & =\int_{\Omega} I(x, t) \mathrm{d} x, \\
\tilde{R}(t) & =\int_{\Omega} R(x, t) \mathrm{d} x, \\
\tilde{N}(t) & =\int_{\Omega}(S+I+R)(x, t) \mathrm{d} x .
\end{aligned}
$$

For these functions the required qualitative properties are as follows (cf. the properties P1-P3 in the previous section).

$\left[\mathrm{P}^{\star}\right]$ (Mass conservation property) The number of individuals does not change in time: $\tilde{N}(t)$ is independent of $t$.

$\left[\mathrm{P2}^{\star}\right]$ (Nonnegativity property) The density functions are nonnegative: condition $S(x, 0), I(x, 0), R(x, 0) \geq 0$ for all $x$ implies $S(x, t), I(x, t), R(x, t) \geq 0$ for all $x$ and $t>0\left(P 2^{\star}\right.$ is the same as $\left.P 2\right)$.

$\left[\mathrm{P}^{\star}\right]$ (Monotonicity property) The number of susceptibles cannot grow and the number of removed cannot decrease in time: $\tilde{S}\left(t_{2}\right) \leq \tilde{S}\left(t_{1}\right)$ and $\tilde{R}\left(t_{2}\right) \geq \tilde{R}\left(t_{1}\right)$ for all $0 \leq t_{1}<t_{2}$.

Theorem 4. The solution of the system (6) possesses the above qualitative properties $P 1^{\star}-P 3^{\star}$.

Proof. In order to show property $\mathrm{P} 1^{\star}$ we take the derivative of $\tilde{N}(t)$.

$$
\tilde{N}^{\prime}(t)=d_{S} \int_{\Omega} \Delta_{D} S(x, t) \mathrm{d} x+d_{I} \int_{\Omega} \Delta_{D} I(x, t) \mathrm{d} x+d_{R} \int_{\Omega} \Delta_{D} R(x, t) \mathrm{d} x
$$

Hence, using the divergence theorem and the homogeneous Neumann boundary condition, we arrive at the relation $\tilde{N}^{\prime}(t)=$ 0 , which yields the first property.

We show property $\mathrm{P}^{\star}$. We rewrite the first two equations of the system (6) in the form

$$
\begin{aligned}
S_{t}^{\prime}(x, t)-d_{S} \Delta_{D} S(x, t) & =F_{1}(S, I), \\
I_{t}^{\prime}(x, t)-d_{I} \Delta_{D} I(x, t) & =F_{2}(S, I),
\end{aligned}
$$

where $F_{1}(S, I)=-k I(x, t) S(x, t)$ and $F_{2}(S, I)=k I(x, t) S(x, t)-b I(x, t)$. Since $F_{1}(0, I) \geq 0$ and $F_{2}(S, 0) \geq 0$, we can use Lemma 14.20 in [14] (see also [15]), which results in the relations $S(x, t) \geq 0$ and $I(x, t) \geq 0$ for any sufficiently smooth 
nonnegative initial functions. Hence, due to the nonnegativity of $I$, the third equation of (6) yields a linear parabolic equation with nonnegative source function for the unknown function $R(x, t)$, therefore it has nonnegative solution, too.

To show property $\mathrm{P}^{\star}$, we use the homogeneous Neumann boundary condition and the nonnegativity property.

$$
\tilde{S}^{\prime}(t)=d_{S} \int_{\Omega} \Delta_{D} S(x, t) \mathrm{d} x-k \int_{\Omega} I(x, t) S(x, t) \mathrm{d} x=-k \int_{\Omega} I(x, t) S(x, t) \mathrm{d} x \leq 0,
$$

which shows that $\tilde{S}(t)$ is non-increasing in time. Analogically, we have the relation

$$
\tilde{R}^{\prime}(t)=\int_{\Omega}\left(d_{R} \Delta_{D} R(x, t)+b I(x, t)\right) \mathrm{d} x=b \int_{\Omega} I(x, t) \mathrm{d} x \geq 0,
$$

which shows that $\tilde{R}(t)$ is monotonically increasing in time. This completes the proof.

Remark 4. We remark that the above statements remain valid if the coefficients $k$ and $b$ are sufficiently smooth nonnegative functions of the space coordinate $x$ and the time coordinate $t$. The proof of the statements is essentially the same as above.

We turn to the finite difference discretizations of the system (6) and give conditions that guarantee the discrete versions of the properties $\mathrm{P} 1^{\star}-\mathrm{P} 3^{\star}$.

\subsection{Properties of the discrete models}

First we construct some discrete models of (6) using finite difference approximations. Similarly to the previous section, we introduce the approximating vectors $s^{n}, i^{n}$ and $r^{n} \in \mathbb{R}^{(M+2)^{D}}$ for the values of the density functions $S, I$ and $R$ at the time instant $t=n \tau$ and in the grid points $\omega_{h}$. Now the values of the density functions are sought also in the boundary points, this is why the approximating vectors are longer than the vectors denoted similarly in the previous section. However, we keep the notations because this will not make any confusion later.

The second order approximation of the $D$-dimensional Laplace operator taking into the account the homogeneous Neumann boundary condition will be denoted by $\bar{Q}_{D} / h^{2}$. The matrix $\bar{Q}_{D}$ can be constructed as follows. Let us define the tridiagonal matrix

$$
\bar{Q}=\left[\begin{array}{ccccc}
-2 & 2 & 0 & \cdots & \\
1 & -2 & 1 & 0 & \cdots \\
& & \cdots & & \\
\cdots & 0 & 1 & -2 & 1 \\
& \cdots & 0 & 2 & -2
\end{array}\right] \in \mathbb{R}^{(M+2) \times(M+2)} .
$$

Then, if $D=1$ then $\bar{Q}_{D}=\bar{Q}$ and in the case of $D=2$ we have $\bar{Q}_{D}=I_{M+2} \otimes \bar{Q}+\bar{Q} \otimes I_{M+2}[16]$.

With the above notations the explicit Euler (EE) scheme can be formulated as follows.

$$
\begin{aligned}
\frac{s^{n+1}-s^{n}}{\tau} & =\frac{d_{s}}{h^{2}} \bar{Q}_{D} s^{n}-k i^{n} \circ s^{n}, \\
\frac{i^{n+1}-i^{n}}{\tau} & =\frac{d_{I}}{h^{2}} \bar{Q}_{D} i^{n}+k i^{n} \circ s^{n}-b i^{n}, \\
\frac{r^{n+1}-r^{n}}{\tau} & =\frac{d_{R}}{h^{2}} \bar{Q}_{D} r^{n}+b i^{n},
\end{aligned}
$$

$n=0,1, \ldots$. The vectors $s^{0}, i^{0}$ and $r^{0}$ are given from the nonnegative initial condition.

A natural choice to approximate the integrals in (21) is the use of the trapezoidal rule. Let us introduce the column vector $\kappa_{D}$ such that $h^{D} \kappa_{D}$ is the vector of the coefficients that multiply the function values in the trapezoidal rule. The coefficients are ordered to a vector in the same way the grid points are ordered. For example, if $D=1$ then

$$
\left(\kappa_{1}\right)_{i}= \begin{cases}1 / 2, & \text { if } x_{i} \text { is one of the endpoints of the interval, } \\ 1, & \text { if } x_{i} \text { is an inner point. }\end{cases}
$$

Then the integrals in (21) can be approximated in the one-dimensional case in the following manner

$$
\int_{0}^{L} S(x, n \tau) \mathrm{d} t \approx h\left(\frac{1}{2} s_{0}^{n}+\sum_{i=1}^{M} s_{i}^{n}+\frac{1}{2} s_{M+1}^{n}\right)=h \kappa_{1}^{T} s^{n} .
$$

If $D=2$ then the vector $\kappa_{2}$ has the form (we use the column-wise one-parameter indexing)

$$
\left(\kappa_{2}\right)_{i}= \begin{cases}1 / 4, & \text { if } x_{i} \text { is a corner point, } \\ 1 / 2, & \text { if } x_{i} \text { is on one of the edges of the square but not a corner, } \\ 1, & \text { if } x_{i} \text { is an inner point. }\end{cases}
$$


Remark 5. Let us notice that $\kappa_{1}^{T} \bar{Q}_{1}$ is equal to the row vector with all zero elements. This can be seen easily from the vector matrix product

$$
\kappa_{1}^{T} \bar{Q}_{1}=[1 / 2,1, \ldots, 1,1 / 2]\left[\begin{array}{ccccc}
-2 & 2 & 0 & \cdots & \\
1 & -2 & 1 & 0 & \cdots \\
& & \cdots & & \\
\cdots & 0 & 1 & -2 & 1 \\
& \cdots & 0 & 2 & -2
\end{array}\right]=[0, \ldots, 0] \in \mathbb{R}^{1 \times(M+2)} .
$$

Similar statement is true in higher dimensions, thus $\kappa_{D}^{T} \bar{Q}_{D}=0$.

Thus, with the above notations the integrals in (21) are approximated and notated at the $n$th time level as follows

$$
\begin{aligned}
& \int_{\Omega} S(x, n \tau) \approx h^{D} \kappa_{D}^{T} s^{n}=: \tilde{s}^{n}, \\
& \int_{\Omega} I(x, n \tau) \approx h^{D} \kappa_{D}^{T} i^{n}=: \tilde{i}^{n}, \\
& \int_{\Omega} R(x, n \tau) \approx h^{D} \kappa_{D}^{T} r^{n}=: \tilde{r}^{n},
\end{aligned}
$$

moreover let $\tilde{N}^{n}=\tilde{s}^{n}+\tilde{i}^{n}+\tilde{r}^{n}$ and $N^{\star}=\tilde{N}^{0}$.

Now we are ready to prove a sufficient condition for the validity of the properties $\mathrm{P}^{\star}-\mathrm{P}^{\star}$ for the EE scheme (27).

Theorem 5. Let us consider the EE scheme for the system (6) with homogeneous Neumann boundary condition. Property P1* is satisfied without any condition, moreover the condition

$$
\tau \leq \min \left\{\frac{h^{2}}{2 D\left(d_{S}+k N^{\star} h^{2-D}\right)}, \frac{h^{2}}{2 D d_{I}+b h^{2}}, \frac{h^{2}}{2 D d_{R}}\right\}
$$

implies properties $P 2^{\star}-P 3^{\star}$.

Proof. We prove property $\mathrm{P} 1^{\star}$. Summing up the equations in (27) and multiplying by $h^{D} \kappa_{D}^{T}$ from the left and using Remark 5 , we have the identity

$$
\frac{\tilde{N}^{n+1}-\tilde{N}^{n}}{\tau}=h^{D} \kappa_{D}^{T}\left(\frac{d_{S}}{h^{2}} \bar{Q}_{D} s^{n}+\frac{d_{I}}{h^{2}} \bar{Q}_{D} i^{n}+\frac{d_{R}}{h^{2}} \bar{Q}_{D} r^{n}\right)=0
$$

for all $n \in \mathbb{N}$, thus $\tilde{N}^{n}$ is independent of $n, \tilde{N}^{n}=N^{\star}$ for all $n \in \mathbb{N}$.

We show property $\mathrm{P}^{\star}$. We suppose that $s^{n}, i^{n}$, and $r^{n}$ are nonnegative, and we show that under the conditions given in the assumptions of the theorem the nonnegativity is preserved for the next $(n+1)$ th time-level, that is for the vectors $s^{n+1}, i^{n+1}$, and $r^{n+1}$.

From the first equation of (27) we have

$$
\begin{aligned}
s^{n+1} & =s^{n}+d_{S} \frac{\tau}{h^{2}} \bar{Q}_{D} s^{n}-\tau k i^{n} \circ s^{n}=d_{S} \frac{\tau}{h^{2}} \operatorname{offdiag}\left(\bar{Q}_{D}\right) s^{n}+\left(s^{n}+d_{S} \frac{\tau}{h^{2}} \operatorname{diag}\left(\bar{Q}_{D}\right) s^{n}-\tau k i^{n} \circ s^{n}\right) \\
& \geq s^{n}+d_{S} \frac{\tau}{h^{2}} \operatorname{diag}\left(\bar{Q}_{D}\right) s^{n}-\tau k i^{n} \circ s^{n}=s^{n}-2 D d_{s} \frac{\tau}{h^{2}} s^{n}-\tau k i^{n} \circ s^{n}=\left(1-2 D d_{s} \frac{\tau}{h^{2}}-\tau k i^{n}\right) \circ s^{n} \\
& \geq\left(1-2 D d_{s} \frac{\tau}{h^{2}}-2 \tau k D \frac{N^{\star}}{h^{D}}\right) \circ s^{n}=\left(1-\frac{2 \tau D}{h^{2}}\left(d_{S}+k N^{\star} h^{2-D}\right)\right) \circ s^{n} \geq 0 .
\end{aligned}
$$

Here we used the structure of the elements of $\bar{Q}_{D}$, the nonnegativity of the vectors $s^{n}, i^{n}$ and $r^{n}$, the estimation

$$
\max i^{n} \leq 2 D \kappa_{D}^{T} i^{n}=2 D \frac{\tilde{i}^{n}}{h^{D}} \leq 2 D \frac{N^{\star}}{h^{D}}
$$

and the first condition for $\tau$ in (29).

Similarly, from the second equation of (27) we obtain

$$
\begin{aligned}
i^{n+1} & =i^{n}+d_{I} \frac{\tau}{h^{2}} \bar{Q}_{D} i^{n}+\tau k i^{n} \circ s^{n}-\tau b i^{n}=d_{I} \frac{\tau}{h^{2}} \operatorname{offdiag}\left(\bar{Q}_{D}\right) i^{n}+\left(i^{n}+d_{I} \frac{\tau}{h^{2}} \operatorname{diag}\left(\bar{Q}_{D}\right) i^{n}+\tau k i^{n} \circ s^{n}-\tau b i^{n}\right) \\
& \geq i^{n}-2 D d_{I} \frac{\tau}{h^{2}} i^{n}+\tau k i^{n} \circ s^{n}-\tau b i^{n} \geq i^{n}-2 D d_{I} \frac{\tau}{h^{2}} i^{n}-\tau b i^{n}=\left(1-\tau \frac{2 D d_{I}+b h^{2}}{h^{2}}\right) \circ i^{n} \geq 0
\end{aligned}
$$

due to the second condition for $\tau$ in (29). 
Analogically, we have

$$
r^{n+1}=r^{n}+d_{R} \frac{\tau}{h^{2}} \bar{Q}_{D} r^{n}+\tau b i^{n} \geq\left(1-\tau \frac{2 D d_{R}}{h^{2}}\right) \circ r^{n} \geq 0
$$

due to the third condition for $\tau$ in (29). Then, the nonnegativity preservation follows by induction.

It is left the proof of property $\mathrm{P}^{\star}$. This can be proved after multiplying the first and the third equations in (27) with the vector $h^{D} \kappa_{D}^{T}$ from the left and using Remark 5. The first equation yields

$$
\frac{\tilde{s}^{n+1}-\tilde{s}^{n}}{\tau}=-k h^{D} \kappa^{T}\left(s^{n} \circ i^{n}\right) \leq 0
$$

due to the nonnegativity of $s^{n}$ ans $i^{n}$. This shows that $\tilde{s}^{n}$ is a nonincreasing function of $n$.

From the third equation

$$
\frac{\tilde{r}^{n+1}-\tilde{r}^{n}}{\tau}=b h^{D} \kappa^{T} i^{n} \geq 0
$$

which estimate shows that $\tilde{r}^{n}$ is nondecreasing in $n$.

This completes the proof.

In this part we construct another discretization based on the IMEX-type approximation. Namely, the diffusion part in the model is approximated by the implicit Euler method and the rest by the explicit Euler scheme. Thus, we consider the scheme

$$
\begin{aligned}
& \frac{s^{n+1}-s^{n}}{\tau}=\frac{d_{s}}{h^{2}} \bar{Q}_{D} s^{n+1}-k i^{n} \circ s^{n}, \\
& \frac{i^{n+1}-i^{n}}{\tau}=\frac{d_{I}}{h^{2}} \bar{Q}_{D} i^{n+1}+k i^{n} \circ s^{n}-b i^{n}, \\
& \frac{r^{n+1}-r^{n}}{\tau}=\frac{d_{R}}{h^{2}} \bar{Q}_{D} r^{n+1}+b i^{n},
\end{aligned}
$$

which is an implicit scheme. Hence, to step to the new time level, we must solve systems of linear algebraic equations. This price must be paid for less strict conditions to preserve the qualitative properties.

In the proof of the next theorem, we will use the M-matrix technique. A square matrix $A$ is an M-matrix if its offdiagonal elements are nonpositive and there exists a positive vector $g$ such that $A g$ is also positive. It is known that M-matrices are invertible and their inverses are nonnegative [17].

Theorem 6. Let us consider the IMEX scheme for the system (6) with homogeneous Neumann boundary condition. Property P ${ }^{\star}$ is satisfied without any condition, moreover the condition

$$
\tau \leq \min \left\{\frac{h^{D}}{2 k D N^{\star}}, \frac{1}{b}\right\}
$$

implies properties $P 2^{\star}-P 3^{\star}$.

Proof. Property $\mathrm{P} 1^{\star}$ can be verified in the same way as in the previous proof.

Let us rewrite system (36) as follows

$$
\begin{aligned}
& \left(I_{(M+2)^{D}}-\frac{d_{S} \tau}{h^{2}} \bar{Q}_{D}\right) s^{n+1}=\left(1-\tau k i^{n}\right) \circ s^{n}, \\
& \left(I_{(M+2)^{D}}-\frac{d_{I} \tau}{h^{2}} \bar{Q}_{D}\right) i^{n+1}=\left(1+\tau k s^{n}-\tau b\right) \circ i^{n}, \\
& \left(I_{(M+2)^{D}}-\frac{d_{R} \tau}{h^{2}} \bar{Q}_{D}\right) r^{n+1}=r^{n}+\tau b i^{n} .
\end{aligned}
$$

The matrices on the left hand sides are M-matrices. This can be seen easily if we choose the vector $g$ with the elements $g_{i}=1$ and noticing that the row sums of $\bar{Q}_{D}$ equal zero. This fact implies that the iteration can be executed and that to show property $\mathrm{P}^{\star}$ it is enough to show the nonnegativity of the vectors on the right hand sides assuming that $s^{n}, i^{n}$ and $r^{n}$ are nonnegative. The first vector is nonnegative because

$$
1-\tau k i^{n} \geq 1-2 \tau k D \frac{N^{\star}}{h^{D}} \geq 0,
$$




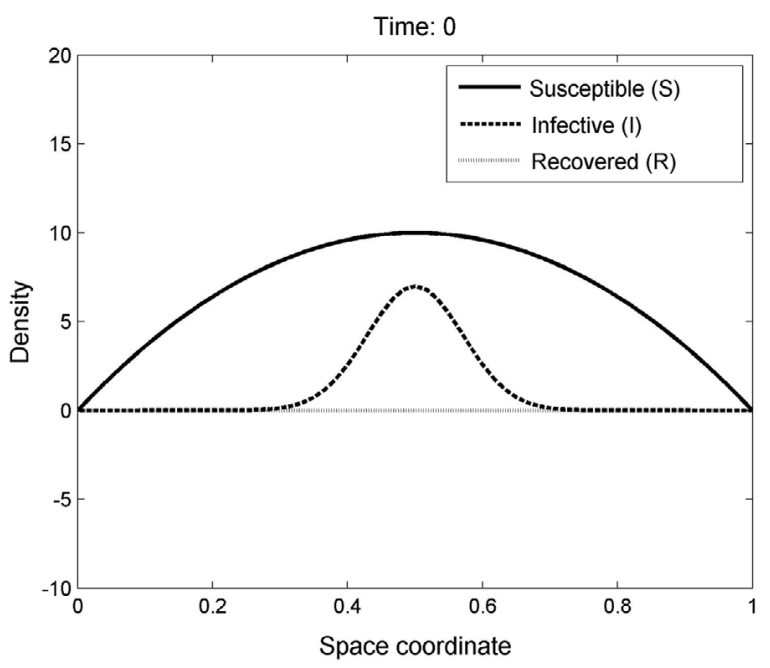

Fig. 1. Initial density functions in the one-dimensional lsSIR model.

where we used the estimate (31) and the assumption (37) of the theorem for $\tau$. The nonnegativity of the second vector follows from the estimate

$$
1+\tau k s^{n}-\tau b \geq 1-\tau b \geq 0
$$

in view of (37). The nonnegativity of the third vector is trivial. Thus property $\mathrm{P}^{\star}$ is satisfied.

Since $\kappa_{D}^{T} \bar{Q}_{D}$ is the zero vector (Remark 5), the proof of property P3* is exactly the same as in the previous proof.

\section{Numerical tests}

In this section we verify the results obtained for adequate time step choices in the previous sections. The condition obtained for the time step guarantees the stability of the numerical methods. This can be seen in the lsSIR case from the estimate $s^{n}+i^{n}+r^{n} \leq N_{\max }$. In the dsSIR case, the stability is ensured by the estimate (31) and by similar estimates for the vectors $s^{n}$ and $r^{n}$.

\subsection{Tests for the lsSIR model}

First we choose a one-dimensional model with the parameter choice $b=0.03$ in the form (4) with homogeneous Dirichlet boundary condition. We solve the problem on the interval $[0,1](L=1)$ and the radius of the infection is set to $\delta=0.01$. With this choice we have $\vartheta=\delta$ and $\varphi=\delta^{3} / 12$. The spatial step size is set to $h=1 / 60(M=59)$. The initial population densities are seen in Fig. 1. For these initial functions we have $N_{\max }=17$.

For the EE method, condition (12) requires the upper bound $\tau \leq 5.5494$. We test the qualitative properties with the time step values: $\tau=5$ (below the bound) and $\tau=15$ (above the obtained bound) and compute the solution at the time level $t=30$. The numerical solutions can be seen in Fig. 2. We can see that the solution that applies the time step $\tau=15$, thus above the obtained bound, does not fulfil the required qualitative properties. The values of the density function of the susceptible population are negative around the space coordinate $x=0.5$. The solution obtained with the time step $\tau=5$ shows a qualitatively adequate form.

Because $h^{\star}=0.0040825$, condition (17) results in no upper bound for the time step in the case of the IMEX method. The solution will be qualitatively correct for all time steps.

The simulation process shows that two waves of the infectious subpopulation moves from the middle of the interval in the directions of the two ends of the interval. The wavelike motion of the infection can happen because the density of the susceptible subpopulation is high enough. The wave can move until the density of the susceptibles drops below the threshold $S^{\infty} \leq b / \vartheta($ see (10)). The left panel of Fig. 3 shows the solution at the time level $t=260$. The left hump in the dashed line of the infectives moves to the left and the other hump moves to the right. The right panel shows the final stationary state after the infectious wave dies out because of the insufficient number of susceptible members.

Now we turn to a $2 \mathrm{D}$ problem with the setting $b=0.05, \delta=0.07, W(u)=10 \max \{0,1-u / \delta\}$. With these choices we have $\varphi=10 \delta^{4} \pi / 40, \vartheta=10 \delta^{2} \pi / 3$. In the numerical solution we use $M=40$ inner points in each coordinate direction. The initial functions can be seen in Fig. 4. With these initial functions we have $N_{\max } \leq 18$. 

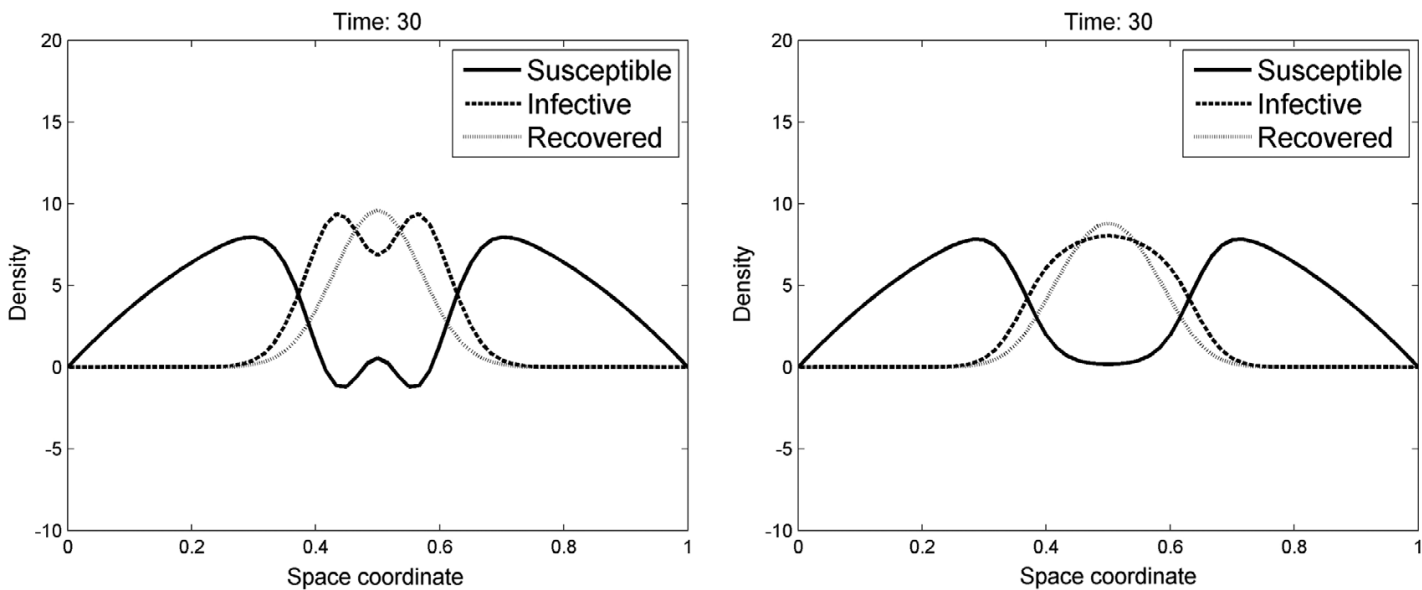

Fig. 2. Numerical solutions at the time level $t=30$ with the time steps $\tau=15$ and $\tau=5$.
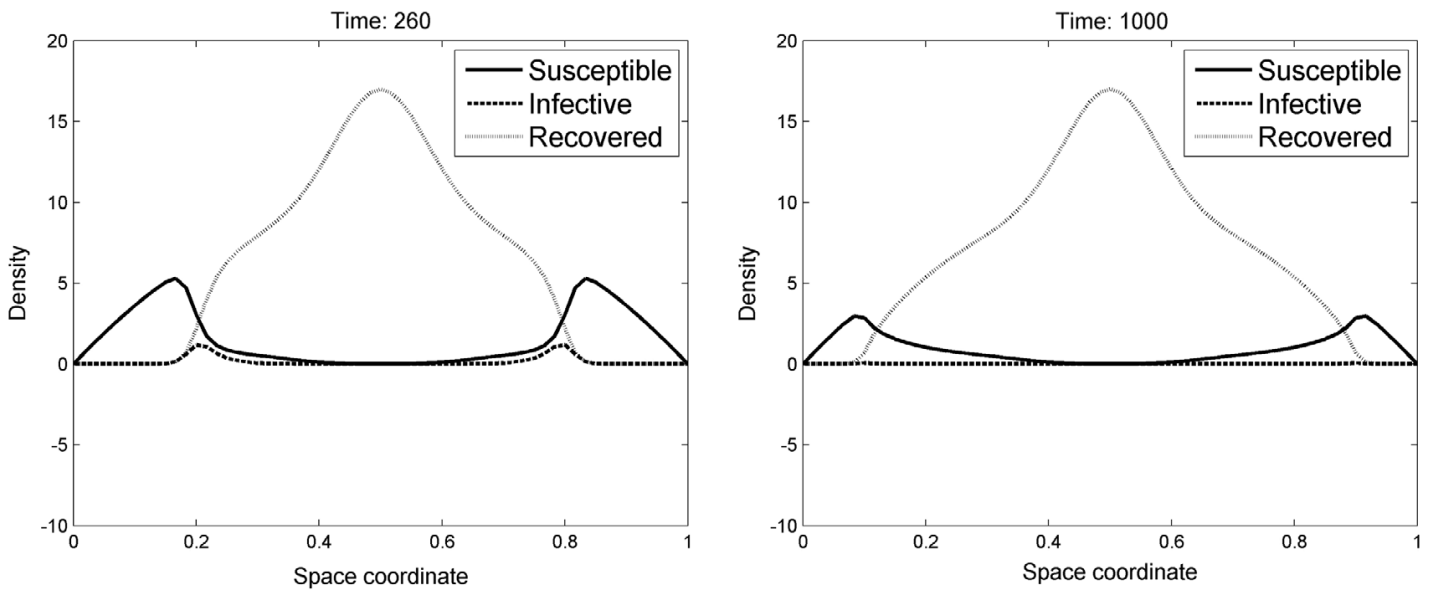

Fig. 3. Numerical solutions at the time levels $t=260$ and $t=1000$ (final stationary solution) that show moving wave front of the infective subpopulation.
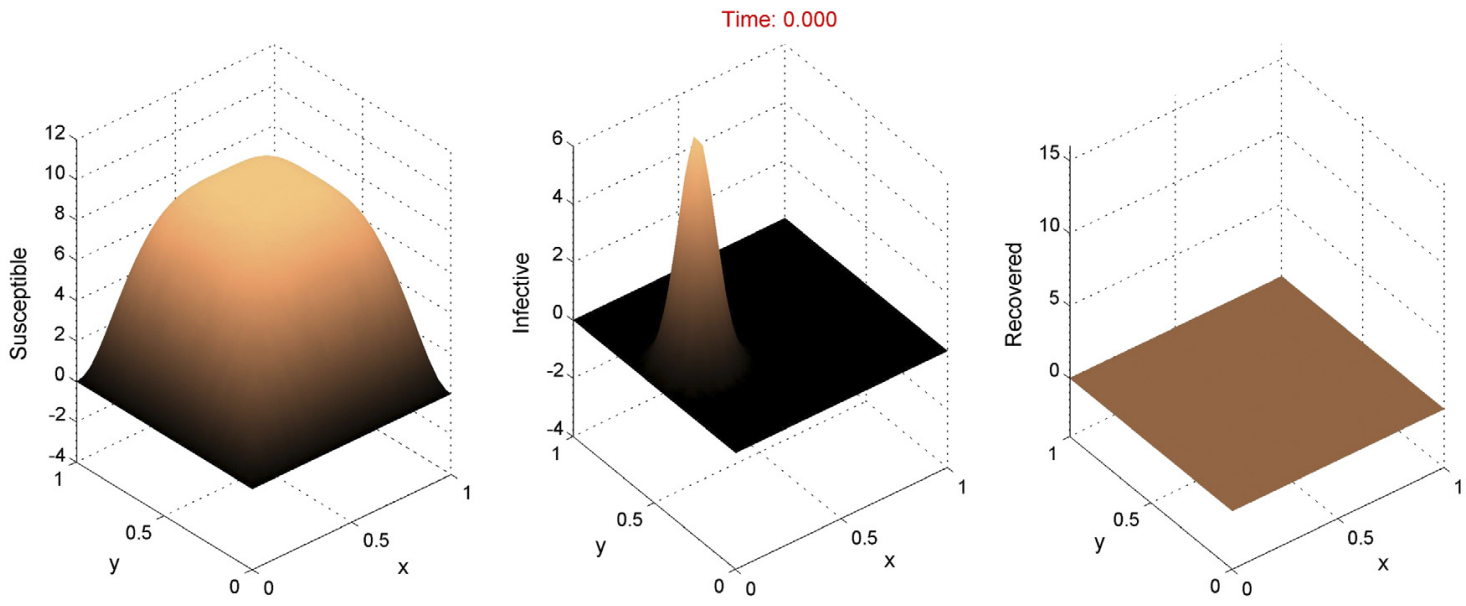

Fig. 4. The initial density functions in the two-dimensional lsSIR model. 

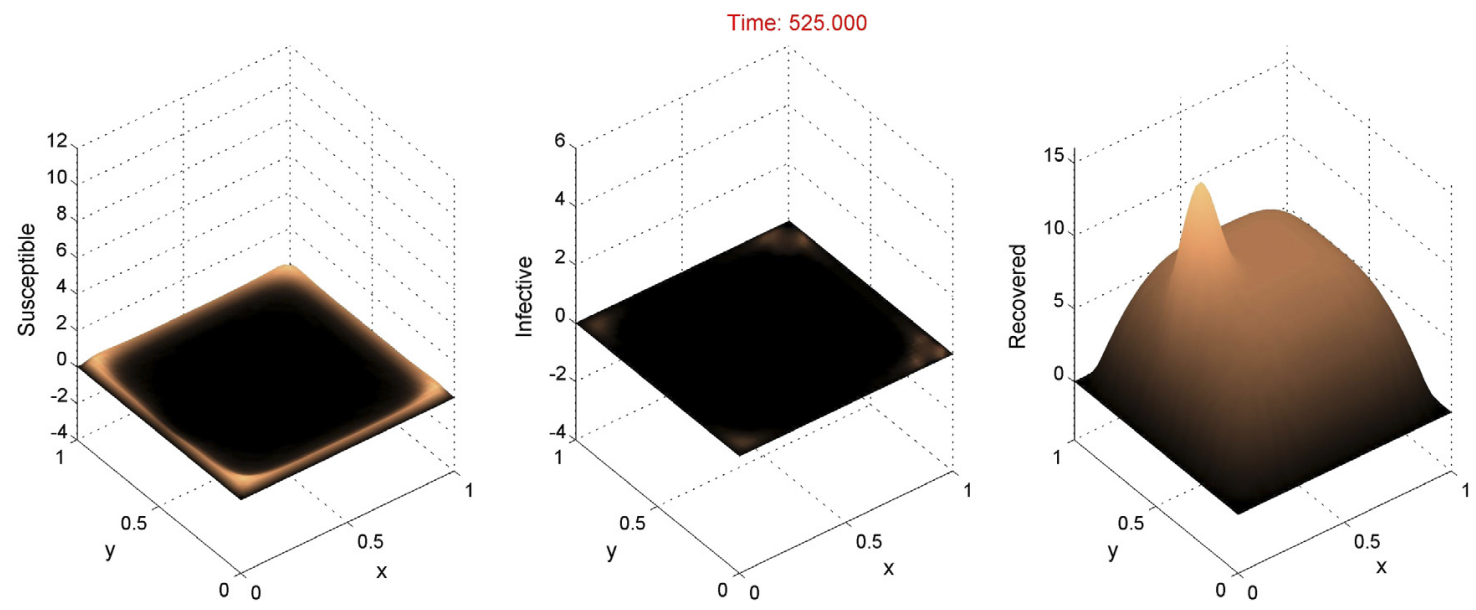

Fig. 5. The final stationary density functions in the two-dimensional lsSIR model.

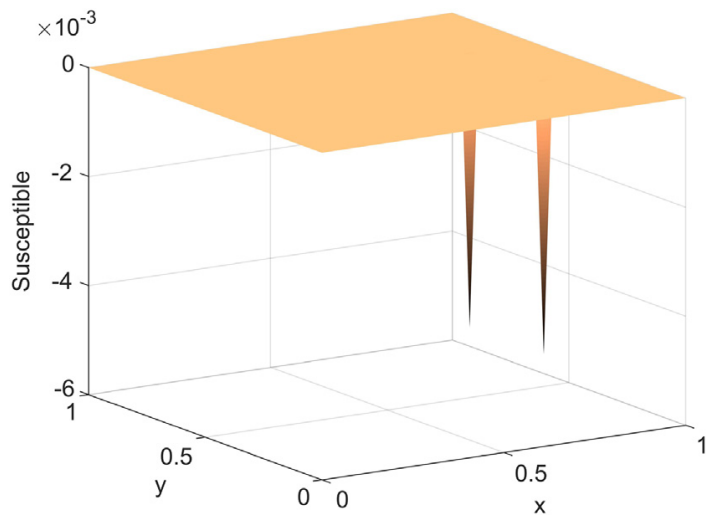

Fig. 6. The negative part of $s^{22}-s^{23}$ (reshaped to the unit square). The figure shows that at certain points the density function of the susceptibles increases.

Now $h=0.02439<h^{\star}=0.03834$, thus the upper bound for the time step is $\tau \leq 0.7360$ in view of Theorem 3. When we apply a time step below this threshold, say $\tau=0.7$, the solution is qualitatively correct and we obtain the final stationary solutions seen in Fig. 5 . If we choose the time step $\tau=2.1$, thus above the time step bound, the final stationary solution will be the same. However in certain time steps the monotonicity property does not hold. For example, the vector $s^{22}-s^{23}$ (thus the difference between the density of the susceptibles at the time levels $t=46.2$ and $t=48.3$ ) must be nonnegative because $s^{n}$ is monotonically decreasing in $n$. If we plot (reshaped to the unit square) the negative part of the difference of these two vectors we should get the constant zero function. In contrast, we obtain for this negative part the graph in Fig. 6. Wave form solution can occur in this two-dimensional model too. (See Fig. 7.) The epidemic wave moves to the positive $x$-direction. At the corners some susceptibles will be left because the density of the susceptibles is not enough to conduct an epidemic wave close to the corners. The necessary lower bound for the density of the susceptibles is $b / \vartheta=0.9744$.

\subsection{Tests for the dsSIR model}

We solve problem (6) on the unit square with homogeneous Neumann boundary condition. The parameters are set as $k=0.1, d=0.01$ and $b=0.07$. We use a spatial mesh with $M=10$ inner points in each direction. The initial functions can be seen in Fig. 8.

With these initial functions we have $N^{\star}=10.9387$. We apply the IMEX scheme because it gives the bigger upper bound for the time step, namely we have $\tau \leq 0.00188882$ (see Eq. (37)). With the time steps below the above bound, the solution is qualitatively adequate. When we enlarge the value of $\tau$, for example to a relatively high value $\tau=3$ then we obtain the final stationary solutions seen in Fig. 9. However, this final solution is reached through some qualitatively inadequate states. For example, on the second time level $(t=6)$, the density of the susceptible population is negative in certain points. These values have not practical meaning. The above numerical tests verify our theoretical results. 

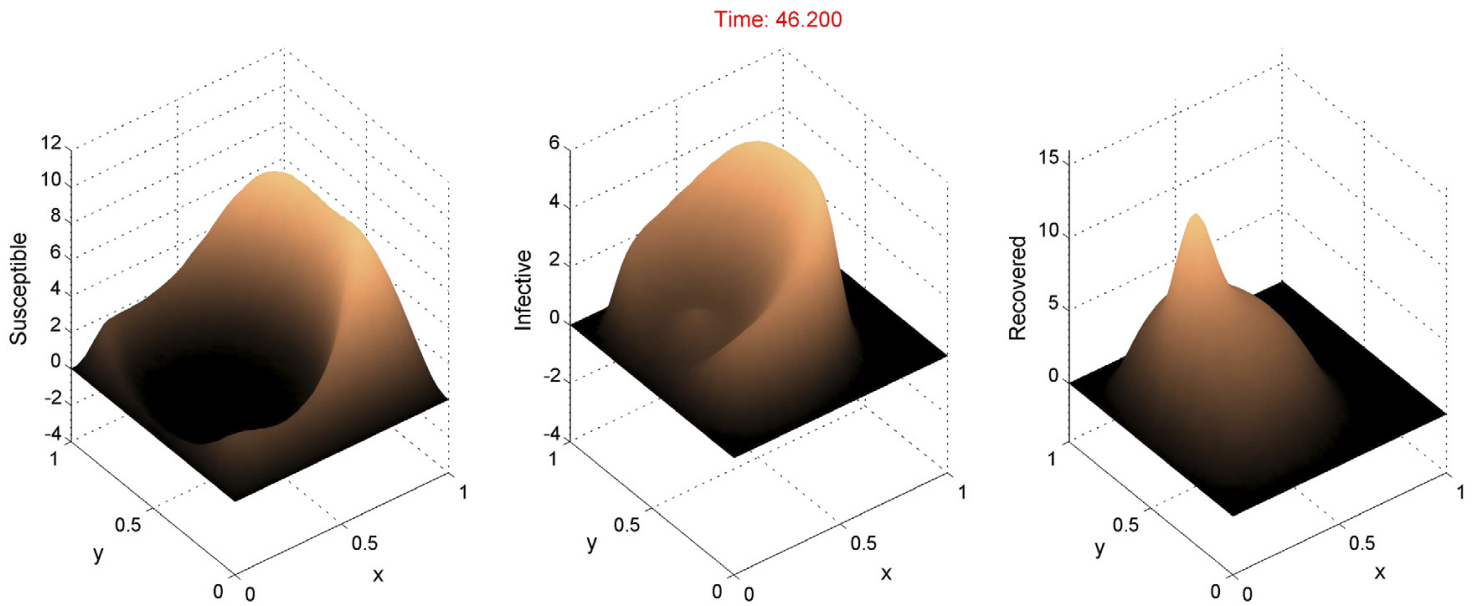

Fig. 7. The moving wave front in the lsSIR model.
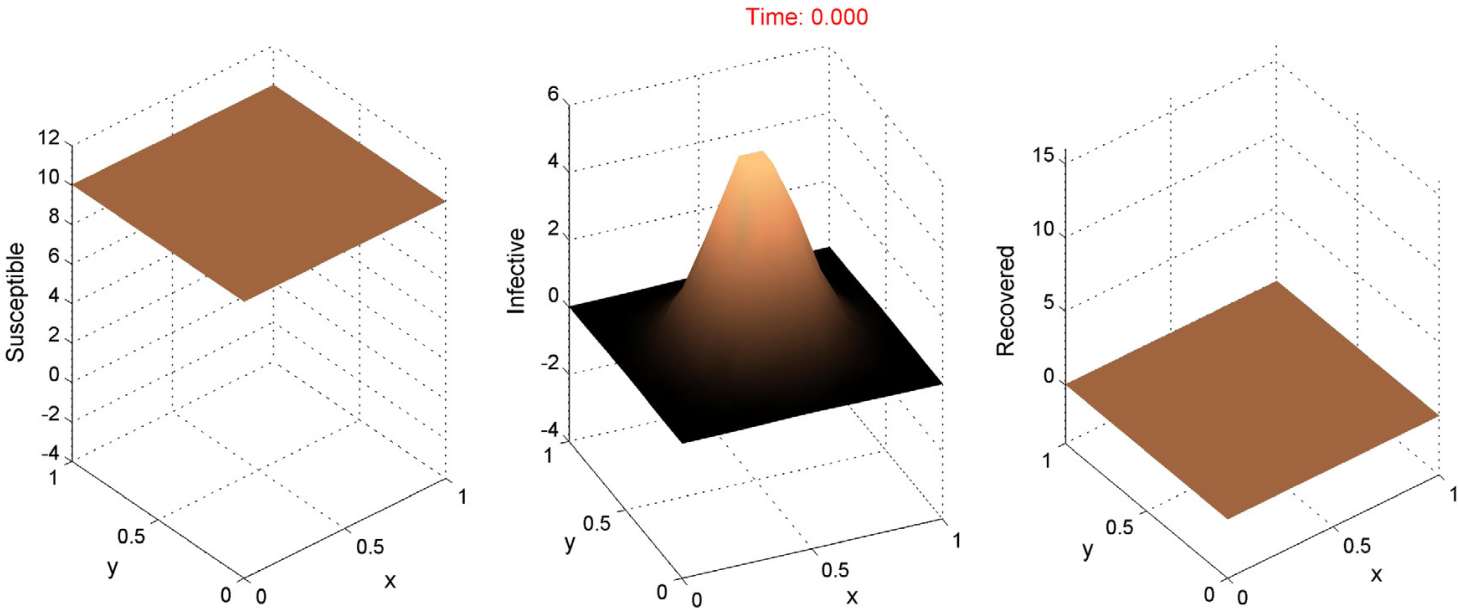

Fig. 8. The initial functions for the dsSIR model.
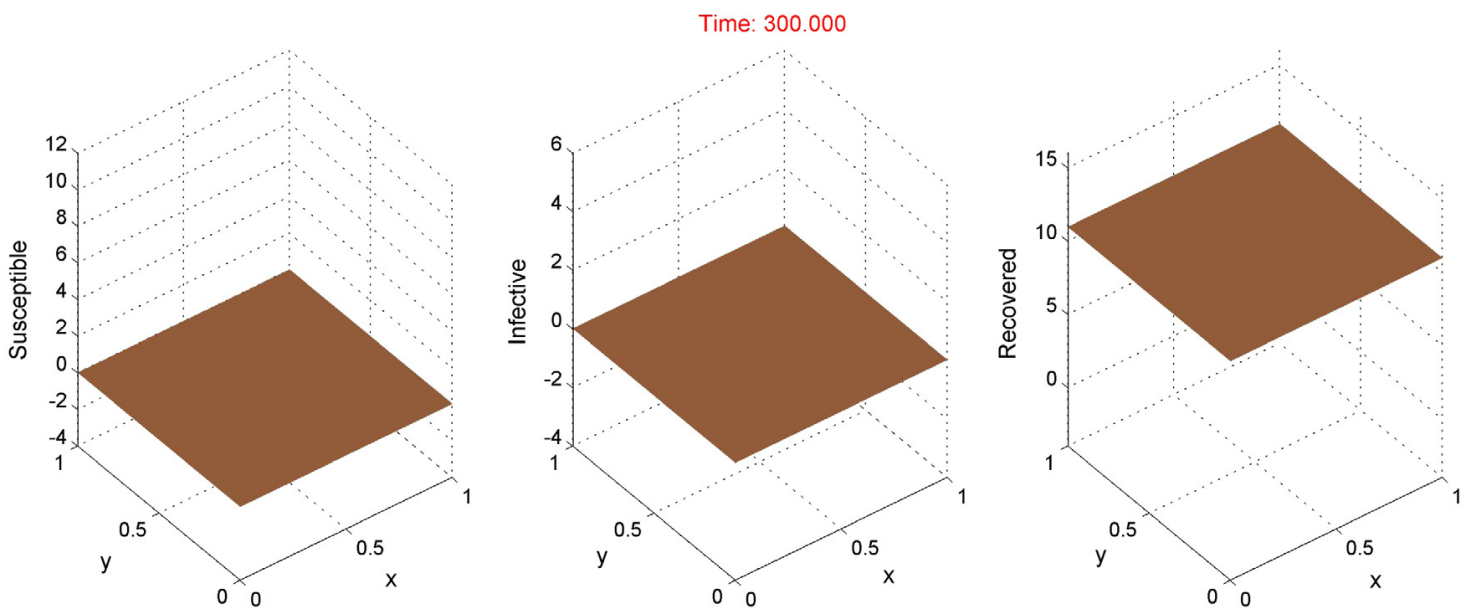

Fig. 9. The final stationary solutions for the dsSIR model. 


\section{Conclusions, future plans}

We saw that it is possible to solve the IsSIR and dsSIR models of spatial disease propagation in a qualitatively adequate manner. If we choose the time step according to some upper bounds then the numerical solution will mirror the basic qualitative properties of the original disease propagation process. Theorems 2, 3, 5 and 6 tell us how to choose the time steps adequately. In the case when the mesh parameters $\tau$ and $h$ tend to zero then the conditions result in upper bounds for the Courant number $\tau / h^{2}$. In the particular case of the EE method in the dsSIR model with spatial dimension $D \geq 3$, we have upper bound for the ratio $\tau / h^{D}$.

A possible continuation of the work presented in this paper is to extend the results to finite element methods. That methods are able to handle more realistic landscapes with more complicated geometry. An interesting extension can be the application of the models to some known epidemic cases, such as the black death in the middle of the 13th century, to compare the predicted population values with the historical ones.

\section{Acknowledgement}

This research was supported by the Hungarian Scientific Research Fund OTKA, No. 112157.

\section{References}

[1] R.M. Anderson, Population Dynamics of Infectious Diseases: Theory and Applications, Chapman and Hall, London-New York, 1982.

[2] F. Brauer, C. Castillo-Chávez, Mathematical Models in Population Biology and Epidemiology, Springer, New York, 2001.

[3] V. Capasso, Mathematical Structures of Epidemic Systems, in: Lecture Notes in Biomathematics, vol. 97, Springer, 2008.

[4] D.S. Jones, B.D. Sleeman, Differential Equations and Mathematical Biology, in: Chapman \& Hall/CRC Mathematical Biology and Medicine Series, CRC Press, 2011.

[5] W.O. Kermack, A.G. McKendrick, A contribution to the mathematical theory of epidemics, Proc. R. Soc. A: Math. Phys. Eng. Sci. 115 (772) (1927) $235-240$.

[6] D.G. Kendall, Mathematical models of the spread of infection, in: Mathematics and Computer Science in Biology and Medicine, H.M.S.O, London, 1965, pp. 213-225.

[7] F. van den Bosch, J. Metz, J. Zadoks, Pandemics of focal plant disease, a model, Phytopathology 89 (1999) 495-505.

[8] L.A. Meyers, B. Pourbohloul, M.E.J. Newman, D.M. Skowronski, R.C. Brunham, Network theory and SARS: predicting outbreak diversity, J. Theoret. Biol. 232 (2005) 71-81.

[9] M.E.J. Newman, Spread of epidemic disease on networks, Phys. Rev. E 66 (2002) 016128.

[10] S. Chinviriyasit, W. Chinviriyasit, Numerical modelling of an SIR epidemic model with diffusion, Appl. Math. Comput. 216 (2010) $395-409$.

[11] M. Kolev, M. Koleva, L. Vulkov, Two positivity preserving flux limited, second-order numerical methods for a haptotaxis model, Numer. Methods Partial Differential Equations 29 (4)(2013) 1121-1148.

[12] I. Faragó, R. Horváth, On a spatial epidemic propagation model, in: G. Russo, V. Capasso, G. Nicosia, V. Romano (Eds.), Progress in Industrial Mathematics At ECMI 2014 (Taormina, Italy, June 2014), Springer, ISBN: 978-3-319-23412-0, 2017.

[13] I. Faragó, R. Horváth, On some qualitatively adequate discrete space-time models of epidemic propagation, J. Comput. Appl. Math. 293 (2016) $45-54$.

[14] J. Smoller, Shock Waves and Reaction-Diffusion Equations, Springer, 1983.

[15] E. Lotfi, M. Maziane, et al., Partial differential equations of an epidemic model with spatial diffusion, Int. J. Partial Differential Equations 2014 (2014) $1-6$.

[16] J.W. Thomas, Numerical Partial Differential Equations, Finite Difference Methods, in: Texts Is Applied Mathematics, vol. 22, Springer, 1995.

[17] A. Berman, R.J. Plemmons, Nonnegative Matrices in the Mathematical Sciences, SIAM, 1994. 